\title{
The Effect of Induced Intraocular Straylight on Perimetric Tests
}

\author{
Ciara Bergin, ${ }^{1}$ Tony Redmond, ${ }^{2,3}$ Neil Nathwani, ${ }^{1}$ Gay Mary Verdon-Roe, ${ }^{2}$ \\ David P. Crabb, ${ }^{1}$ Roger S. Anderson, ${ }^{2,3}$ and David F. Garway-Heath ${ }^{1}$
}

Purpose. To investigate the effect of intraocular straylight (IOS) on threshold measurements made by four types of perimetry: standard automated perimetry (SAP), frequency-doubling technology (FDT) perimetry, flicker-defined form (FDF) perimetry, and the Moorfields Motion Displacement Test (MDT).

Methods. Six healthy young (21-29 years) psychophysically experienced observers were tested five times with each perimeter, each time with one of five different white opacity filters (WOFs), to induce various levels of IOS. Each WOF gave rise to an increase in IOS, measured with a straylight meter, of between $10 \%$ and $200 \%$ from baseline and ranging in effect from normal ageing to significant cataract. The change in sensitivity from baseline was normalized, allowing comparison of standardized $(z)$ scores (change divided by the standard deviation of normative values) for each instrument.

Results. Average SAP, FDT, and FDF thresholds were significantly affected $(P<0.001)$ by moderate to large increases in IOS (50\%-200\%), whereas MDT remained largely unaffected over the same range of straylight levels

Conclusions. The Moorfields MDT shows greater resilience to the effects of additional straylight compared with SAP, FDT, or FDF. (Invest Ophthalmol Vis Sci. 2011;52:3676-3682) DOI: 10.1167/iovs.10-6642

A utomated static perimetry has become a well-established and valuable tool for the detection and monitoring of sensitivity loss that accompanies various diseases of the visual pathway, especially glaucoma. Although the structure-function debate continues, no consensus has been reached on why, in some individuals, structural loss is observed at the retinal level before functional loss is identified with standard auto-

From the ${ }^{1}$ Department of Optometry and Visual Sciences, City University London, London, United Kingdom; the ${ }^{2}$ NIHR (National Institute of Health Research) Biomedical Research Centre for Ophthalmology, Moorfields Eye Hospital NHS (National Health Service) Foundation Trust and UCL Institute of Ophthalmology, London, United Kingdom; and the ${ }^{3}$ Vision Science Research Group, School of Biomedical Sciences, University of Ulster, Coleraine, Northern Ireland, United Kingdom.

Supported by the Department of Health's National Institute for Health Research (NIHR) Biomedical Research Centre for Ophthalmology at Moorfields Eye Hospital NHS Foundation Trust and the UCL Institute of Ophthalmology (DFGH). DFGH's chair at UCL is supported by funding from the International Glaucoma Association.

Submitted for publication September 27, 2010; accepted December 8,2010 .

Disclosure: C. Bergin, P; T. Redmond, None; N. Nathwani, None; G.M. Verdon-Roe, P; D.P. Crabb, P; R.S. Anderson, None; D.F. Garway-Heath, Carl Zeiss Meditec (F, C, R), Heidelberg Engineering $(\mathrm{F}, \mathrm{R})$, OptoVue $(\mathrm{F}, \mathrm{R}), \mathrm{P}$

Corresponding author: David P. Crabb, Department of Optometry and Visual Sciences, City University London, Northampton Square, London EC1V 0HB, UK; david.crabb.1@city.ac.uk. mated (contrast) perimetry (SAP) with a white-on-white stimulus. ${ }^{1-3}$ This question has prompted a search for newer perimetric techniques designed to detect functional changes earlier or to be more robust to factors that affect the measurement of retinal function. ${ }^{4-10}$ The seemingly low diagnostic sensitivity of perimetry is due, to a large degree, to inherent variability within the normative data and measurement noise. ${ }^{5}$ With age being an important risk factor for glaucoma, it is imperative that other causes of age-related losses of vision (particularly optical deficits) be accounted for when quantifying neural loss due to disease.

There have been many studies demonstrating the effect of cataract on visual field sensitivity, mainly by comparing visual function before and after cataract extraction and intraocular lens implantation. Several of these studies demonstrated that $\mathrm{SAP}^{6-8}$ and frequency-doubling technology $(\text { FDT })^{9,10}$ mean deviations were significantly reduced in the presence of cataract. Although it is recognized that cataract can significantly affect perimetric thresholds, the degree and nature of lens opacity or cataract was poorly quantified or characterized in these studies. In addition, it is not immediately clear which particular aspects of optics confound the quantification of neural vision loss to the greatest degree. The ageing lens displays changes in general light absorption, ${ }^{11,12}$ forward and backward light scatter, ${ }^{13}$ and spectral transmission characteristics. ${ }^{14-16}$ The relative contribution of each of these to the increase in perimetric threshold is not clearly understood.

One result of ageing in general, and cataract formation in particular, is an increase in intraocular straylight (IOS), arising from increased forward light scatter. ${ }^{17,18}$ Increases in IOS cause a shortening and widening of the point-spread function of the eye, resulting in reduced contrast sensitivity. ${ }^{17}$ Although IOS varies between individuals, even in the young healthy eye, largely as a result of differing ocular pigmentation, ${ }^{19}$ IOS values associated with cataract are greater. ${ }^{20,21}$ It is reasonable to expect that some perimetric stimuli would be more susceptible to the influence of additional IOS than others, as some are being modulated in contrast, whereas others are being modulated along a spatial scale. It follows that of the various forms of commercially available perimeters (which employ different stimulus types with different configurations) some may be affected more than others with additional IOS.

Investigators in several studies have used white diffusing filters during perimetry to simulate the effects of cataract. ${ }^{20,22-24}$ These studies have shown a general depression of the hill of vision with SAP (on both the Humphrey Field Analyzer; HFA [Carl Zeiss Meditec, Dublin, CA] and the Octopus perimeter [Haag Streit, Köniz, Switzerland]) and FDT perimetry. There were significant reductions in sensitivity after the introduction in front of the eye of diffusing filters consisting of photographic filter foils, ${ }^{22} 500$-nm latex beads, ${ }^{23}$ or ground glass. ${ }^{24}$ The results found with these cataract simula- 
tions were consistent with those reported in cases of pre- and postoperative cataract removal, although the degree of simulated cataract was not quantified in a way that could be correlated with physiological or pathologic states of the eye's optical quality.

Membrey et al. ${ }^{22}$ performed a study to investigate the effects of simulated cataract (using one white diffusing filter) on the FDT (FDT1, 24-2), SAP (HFA II, full threshold), and a prototype Motion Displacement Test (MDT; Moorfield's Eye Hospital, London, UK) (using a single-line presentation). Comparisons were made between these three instruments, using standardized values for the thresholds. They concluded that the MDT stimulus is more resilient to this form of simulated cataract than are the stimuli used in SAP and FDT. Several modifications have been made to the test strategies, formats, and stimuli used in these clinical devices since that study was conducted, and the relationship between the properties of the diffusing filter and the various forms of lens opacity has not been well defined.

Diffusing filters used for cataract simulation have historically been characterized either by an arbitrary number or by their optical density. However, in a recent study, de Wit et al. ${ }^{20}$ considered the use of diffusing filters as a simulation of cataract by comparing the light-scattering properties of the filters with those of cataract. Anderson et al. ${ }^{25}$ recently investigated the effects of IOS induced by white opacity filters (WOFs; LEE Filters, Andover, UK) of different grades on various forms of perimetry: SAP, FDT1, short-wavelength automated perimetry (SWAP), and peripheral grating-resolution perimetry (GRP). The WOFs contain light-scattering particles within the matrix of the polymer, rather than etchings on the surface, resulting in wide-angle scatter similar to that caused by cataract. ${ }^{19,20,25}$ A particular strength of the study by Anderson et al. ${ }^{25}$ was that the magnitude of IOS induced by each filter was quantified using a cataract quantifier (C-Quant; Oculus, Wetzlar, Germany) as described by Franssen et al. ${ }^{26}$

The purpose of this study was to examine the effect of IOS, using methodology similar to that of Anderson et al. ${ }^{25}$ on threshold measurements for stimuli used in four perimeters: HFAII (Carl Zeiss Meditec), the FDT Matrix (Carl Zeiss Meditec), the newly-introduced Heidelberg Edge Perimeter (HEP; Heidelberg Engineering, Heidelberg, Germany), and the Moorfields MDT (Moorfields Eye Hospital).

\section{METHODS}

Six healthy, psychophysically experienced volunteers (aged 21-29 [mean, 26] years) participated in the study. None had a history of ocular disease or systemic disease that might affect visual performance, and none had undergone ocular surgery. Inclusion criteria included optic disc rim area classified as within normal limits by Moorfields regression analysis of the Heidelberg Retina Tomograph (HRT II; Heidelberg Engineering, Heidelberg, Germany) and intraocular pressure $<21 \mathrm{~mm} \mathrm{Hg}$. The right eye was tested in all the subjects, and each wore appropriate near correction; refractive error ranged from 0 to -4.25 (mean, -2.10$) \mathrm{D}$ sphere. The subjects had negligible astigmatism ( $\leq 0.50 \mathrm{D}$ cylinder). Best corrected visual acuity for each observer was 20/17 (6/5) or better. All subjects were reliable (false positives, $<20 \%$; false negatives, $<33 \%$; and fixation losses $<33 \%$ ) in two initial practice sessions on each perimeter.

The study had local ethics approval from City University London, with recruitment and experimentation adhering to the tenets of the Declaration of Helsinki; informed consent was obtained from all subjects.

\section{Perimetry Instruments}

Four types of perimetry were used:
HFA II, SITA-Standard 24-2 Program. The system presents an intensity-modulated Goldmann III achromatic stimulus on a 10$\mathrm{cd} / \mathrm{m}^{2}$ achromatic background, with 54 test locations. The threshold is the lowest luminance at which the stimulus is detectable $50 \%$ of the times it is presented.

FDT Matrix, 24-2 ZEST Program. The program includes a contrast-modulated $5^{\circ}$ sinusoidal grating $(0.25 \mathrm{cyc} / \mathrm{deg})$ stimulus (encased in a square envelope) with a fixed counterphase flicker of $15 \mathrm{~Hz}$ displayed on a screen of background luminance of $100 \mathrm{~cd} / \mathrm{m}^{2}$ (Johnson CA, et al. IOVS 2004; 45:ARVO E-Abstract 3470) ${ }^{27,28}$; there are 54 test locations. The threshold is the contrast level at which the stimulus (doubling) is detected for $50 \%$ of the presentations made at this contrast level.

HEP, 24- 2 ASTA Program. The stimulus is contrast-modulated $5^{\circ}$ patches of $0.34^{\circ}$ (diameter) dots flickering in counterphase to a background of identical dots with a mean luminance of $50 \mathrm{~cd} / \mathrm{m}^{2}$ at 55 test locations. The random dot background has a density of 3.5 dots/ deg. The threshold is the contrast level for which an illusionary edge of a gray circle is perceived for $50 \%$ of the presentation made ${ }^{29}$; the illusion is termed a flicker-defined form (FDF).

Moorfields MDT, Weighted Binary Search Program. Three oscillations of $200 \mathrm{~ms}$ each modulate the displacement of vertical bar stimuli of $85 \%$ Michelson contrast on a $10-\mathrm{cd} / \mathrm{m}^{2}$ achromatic background, ${ }^{30}$ at 32 test locations. The threshold is the discernable displacement detected for $50 \%$ of the presentations made. ${ }^{30}$

Each perimeter has a different stimulus, detection task, and thus scale on which the threshold is measured. SAP sensitivity is given as the $\log$ ratio between the maximum stimulus intensity and the threshold stimulus intensity ( $1 \mathrm{~dB}$ equates to a change of $0.1 \log$ unit of Weber contrast); the dynamic range of the instrument is 0 to $40 \mathrm{~dB}$ (which, in terms of contrast, is $>99 \%$ contrast to $<1 \%$ contrast). FDT also has a contrast-modulated stimulus; Michelson contrast varies between $100 \%$ and $1 \%$, and thresholds are measured on a $\log$ scale ranging from 0 to $38 \mathrm{~dB}(1 \mathrm{~dB}$ equates to a change of $0.05 \log$ units of Michelson contrast). FDF on the HEP is also a contrast-modulated stimulus. Michelson contrast varies between $100 \%$ and $1 \%$ and is measured on a $\log$ scale ranging from 0 to $22 \mathrm{~dB}$. Finally, the MDT stimulus is modulated in displacement distance, with displacements varying between 2 and $40 \mathrm{~min}$ arc. Contrast is fixed at $85 \%$ Michelson contrast.

\section{White Opacity Filters}

Five white resin opacity-containing filters were used (grades 1-5). These WOFs had mean light transmittance spectra ranging from 0.86 (fog 1) to 0.42 (fog 5) as measured with a spectrophotometer (SpectraScan PR-650 Spectra Colorimeter; Photo Research Inc., Chatsworth, CA) directly against an achromatic CRT screen (luminance: 10 $\mathrm{cd} / \mathrm{m}^{2}$; chromaticity coordinates: $\left.x=0.218, y=0.328\right) .{ }^{25}$ When measured under our experimental conditions with the spectrophotometer at eye level, these WOFs had reduced mean light transmittance spectra ranging from 0.86 (fog 1) to 0.54 (fog 5 ), as measured against an achromatic CRT screen (luminance: $10-100 \mathrm{~cd} / \mathrm{m}^{2}$; corresponding to the range of background luminance used by the perimetric instruments examined). For ease of use, the filters were cut and edged into spectacle frames with large-aperture lenses. Surgical tape (Blenderm; $3 \mathrm{M}$, Bracknell, UK) over a blank lens was used to occlude the nontest eye to eliminate the detection of form or movement.

\section{Psychophysical Measurements}

The C-Quant stray light meter (Oculus) was used to determine IOS $(\log (\mathrm{s}))$ values by the "compensation comparison" method. ${ }^{26} \mathrm{~A}$ baseline (no WOF) IOS and an IOS value with each of the filters were recorded in each subject. The order of measurements was randomized between subjects.

To characterize the subjects, IOS values for each subject under each condition are shown in Figure 1A. The C-quant software provides an estimation of the typical age for equivalent IOS that is simulated 
A

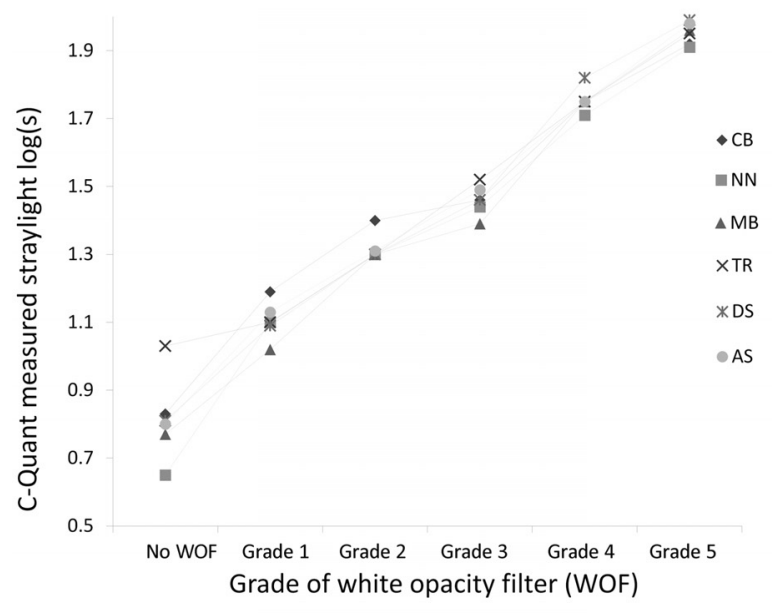

B

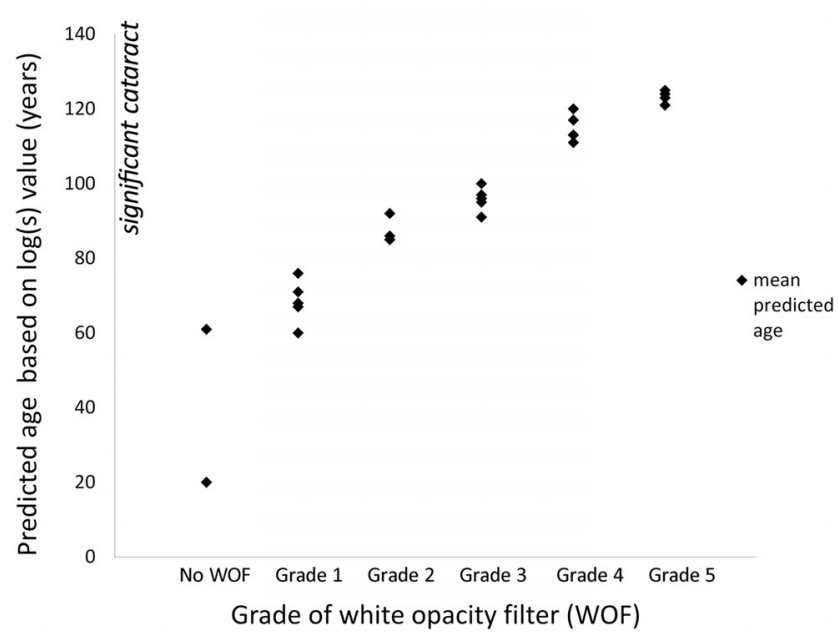

FIGURE 1. (A) The measured change in IOS for each subject at baseline (No WOF) and fog filters 1 to 5 . (B) The age equivalence induced by each WOF across subjects, according to the C-Quant (Oculus) normative database. This normative database gives the mean and the upper and lower limits, which have been translated here into the mean, maximum, and minimum predicted age.

using each WOF. For example, an average 25 -year-old has an IOS value of $\sim 0.85 \mathrm{~dB}$ and an increase in IOS of $40 \%$ (to $1.2 \mathrm{~dB}$ ) renders the subject similar to an average 70 year old. The WOFs yielded an increase in IOS of $10 \%$ to $200 \%$ from baseline, resulting in IOS values seen in normal ageing to those obtained from patients with considerable cataract. Figure $1 \mathrm{~B}$ shows the approximate mean age as estimated by the C-quant normative database with respect to the filters. ${ }^{17,21}$

SAP, FDT perimetry, FDF perimetry, and the MDT were performed once by each subject with each of the five WOFs and once without a filter. The order of the tests and filters was randomized to avoid bias or possible learning effects. Each subject was tested in six sessions, each session consisting of one trial with each perimeter with one (or no) WOF, resulting in a total of 24 trials. After each trial, there was a rest period of 5 minutes; no more than two sessions were performed in 1 day.

\section{Analysis}

Direct comparison of average thresholds is of limited value because each instrument records a measure of sensitivity on a different scale. Therefore, these measures of threshold were normalized for compari- son. A direct comparison between instruments, with respect to the changes in sensitivities due to increases in straylight can be achieved using $z$-scores. ${ }^{31}$ The $z$-scores were derived using the available normative data. To calculate the $z$-score, the difference between the agecorrected mean sensitivity from the normative database at a location and the measured sensitivity value at the matched location were computed. The result was divided by the standard deviation of agecorrected normative values for the location. This transformation established the position of each threshold with respect to the normal ranges (i.e., a value of 1 is $1 \mathrm{SD}$ away from the mean and a value of 1.96 corresponds to the $95 \%$ limit of normality, as shown on the total deviation maps). Values greater than 1.96 were taken to be outside normal limits.

The standardized $z$-score was calculated as the difference from baseline (no WOF) of each sensitivity with each WOF, divided by the standard deviation of age-corrected normative values for the location.

The sources of normative database information were FDF (Flanagan JF, personal communication, July 2010), FDT Matrix (Johnson C, personal communication, July 2010), and the MDT (Moosavi R, personal communication, July 2010). For SAP, a normative database of 120 right eyes of healthy subjects with normal HRT Moorfields regression analysis results and intraocular pressure $\leq 21 \mathrm{~mm} \mathrm{Hg}$ was formed from retrospective data collected at Moorfields Eye Hospital.

All data were then analyzed (MatLab, ver. 7.2.0.232; The MathWorks, Natick, MA). for all 32 matched locations common among the tests.

\section{Results}

The results of a two-way ANOVA with subjects acting as blocks in the experiment and the main factor being each grade of WOF indicated that mean deviation (MD) as measured with FDT, SAP, and FDF worsened across the different WOFs $(P<$ 0.0001 ). Conversely, there was no evidence of a difference in MD for MDT across the different WOFs $(P=0.18)$.

Threshold mean sensitivity (TMS) is the average measured sensitivity for a field test; the relationship between TMS and grade of WOF was examined. It was found that, with a WOF greater than grade 4 , SAP $(P<0.001)$, FDT $(P=0.003)$, and FDF $(P<0.001)$ were significantly affected; MDT TMS values did not have a significant association with the density of WOF filter used ( $P=0.73$; ANOVA).

Each subject had different straylight values for each of the WOF filters used. Therefore, in addition to considering TMS by WOF, TMS was plotted against the measured C-Quant $\log$ (s) values for each subject with each WOF. The results are shown in Figure 2; the shaded area represents the normative range for each instrument. The decibel (or minutes of arc for MDT) loss per $\log (\mathrm{s})$ for each perimeter was estimated by linear regression: the FDF, SAP, and FDT are all significantly associated $(P<$ $0.001)$ with IOS, whereas MDT does not have significant slope $(P=0.34)$. The estimated slopes were SAP, $-1.6 \mathrm{~dB} / \log (\mathrm{s})$; FDT, $-1.7 \mathrm{~dB} / \log (\mathrm{s}) ; \mathrm{FDF},-3.1 \mathrm{~dB} / \log (\mathrm{s})$; and MDT, $-0.1 \mathrm{~min}$ $\operatorname{arc} / \log (\mathrm{s})$.

Figure $3 \mathrm{~A}$ summarizes the $z$-scores (for each subject with each grade of the WOF) against the respective $\log (\mathrm{s})$ values. The results were grouped into three IOS ranges, chosen to correspond loosely with the classification of within normal limits, outside normal limits, and significant cataract given by Franssen et al. ${ }^{26} z$-Scores were derived for every point, and the distribution of these values is summarized in the box-andwhisker plot. These summarize the median (the solid line within the box is the median), the interquartile range (size of the box), and the range of the data points, spanning from the highest and lowest recorded threshold (length of the line).

The average threshold for the subjects did not lie on the average normal line for the various perimeters for the range of 
FIGURE 2. The TMS with respect to IOS, denoted $\log (\mathrm{s})$. The area above (below for Moorfields MDT) the dotted line represents $95 \%$ of the normative range for SAP, FDT, and FDF. It is clear that despite the negative trend for line for SAP, FDT, and FDF, they mainly remain with the normative range of measured values.
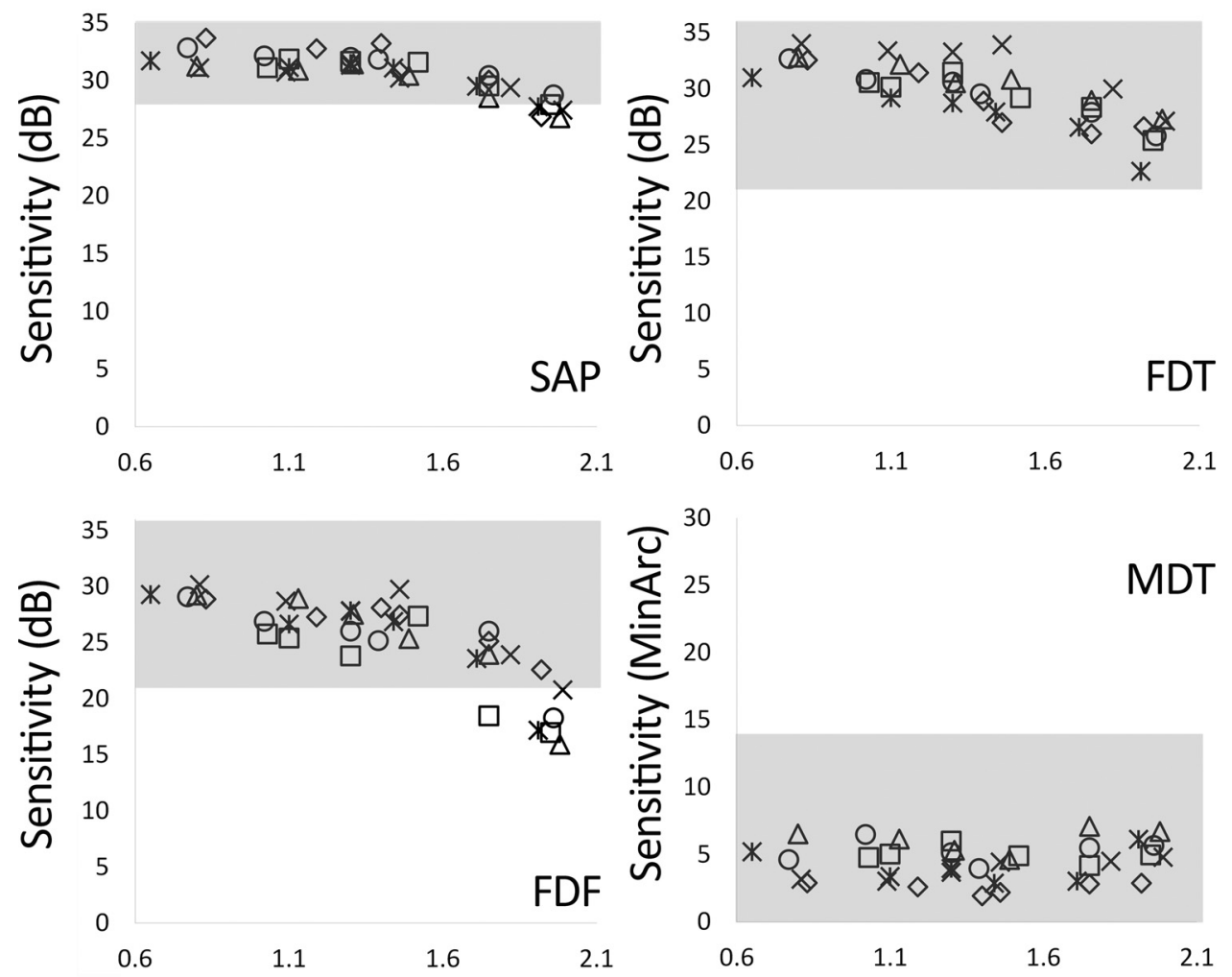

0

MDT

Straylight parameter $\log (\mathrm{s})$

$\diamond \mathrm{CB} \square \mathrm{NN} \triangle \mathrm{MB} \times \mathrm{TR} * \mathrm{DS} \circ \mathrm{AS}$
$10 \%$ to $50 \%$ increase in IOS. For this reason, standardized $z$-scores were derived. In Figure 3B, the standardized $z$-scores are plotted against the percentage increase in straylight. The three bins in this case reflected the percentage change from baseline of IOS: up to $50 \%, 50 \%$ to $100 \%$, and $100 \%$ to $200 \%$. MDT was not significantly affected $(P=0.35, P=0.2$, and $P=$ 0.14 , respectively); this result was consistent across all subjects (data not shown). For increases in IOS of up to 50\%, SAP was not significantly affected $(P=0.37)$, but FDT and FDF were $(P<0.01)$. FDT, FDF, and SAP were all significantly affected, with increases in IOS of greater than $50 \%(P<0.001)$. The box-and-whisker plots summarize the average bias (change induced by the WOFs); the interquartile range (size of the box) describes the consistency of the change in threshold induced by the WOFs across subjects and locations in the field. The spread of the data suggest that MDT has the lowest average, and most consistent, induced change in threshold, and FDF has the highest average, and least consistent, induced change.

Figure 4 shows a plot of the mean standardized $z$-score for each subject and each instrument against the change in straylight. Each symbol represents the mean of the standardized $z$-scores for one subject with one filter; there are four means for each subject and each filter, one for each instrument (four different symbols). The slopes, calculated by linear regression (the intercept was constrained to 0 because normalized $z$ scores were used), give an estimate of the robustness of each of the instruments to increases in IOS. MDT thresholds show little to no association with IOS (slope $=-0.01$ ), SAP shows weak association with IOS (slope $=-1.21, P=0.02$ ), followed with a stronger association by FDT (slope $=-1.32, P<$ 0.01 ) and FDF (slope $=-1.86, P<0.01$ ).

On examining the differences of the effect of IOS on threshold with eccentricity as an explanatory variable, MDT and FDF were uniformly affected by straylight with no significant dif- ferences between the central and peripheral points. For FDT and SAP, significant differences in the degree of effect of IOS $(P<0.05)$ were found between the central and peripheral points.

\section{Discussion}

Perimetry has two purposes: one for monitoring disease and the other for case detection. For both roles, it is important that the test result not be confounded by eye conditions other than the target condition (in this case glaucoma). During monitoring, progressive cataract in patients with stable or suspected glaucoma may result in a change in threshold that may mimic progressive glaucoma, and a diffuse reduction in sensitivity in patients with progressing glaucoma may erroneously be discounted as being due to cataract. For case detection, false-positive results may arise from cataract. Thus, it would be advantageous to have a perimetric test that is robust to the effects of cataract.

An analysis of visual field sensitivity, developed to compensate for the effects of loss of optical quality, is the pattern deviation (PD) map (as in STATPAC for the HFA; Carl Zeiss Meditec). The PD measure partly accounts for diffuse (as with optical) loss by subtracting from the total deviation plot (deviation from the sensitivity of age-matched healthy subjects) the value corresponding to the 85 th percentile of the total deviation measures, thus amplifying focal loss. ${ }^{29}$ However, this diffuse loss is not individualized with respect to the expected reduction in sensitivity due to loss of optical quality. It is a post hoc analysis based only on the measured sensitivity. For this reason, when diffuse loss is accounted for with summary measures such as PSD in the HFA, diffuse glaucomatous loss is hidden. Thus, although PD plots may be useful, in that they help identify sites of focal loss, they remain of limited value. 


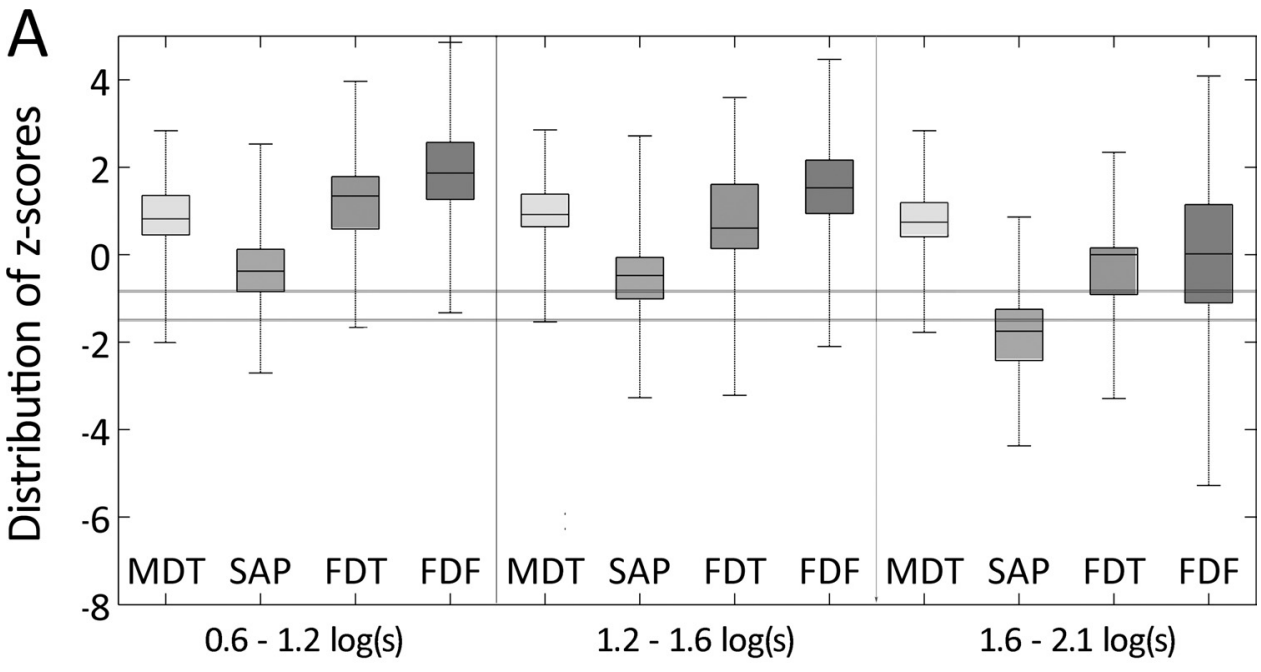

B

\section{Straylight parameter log(s)}

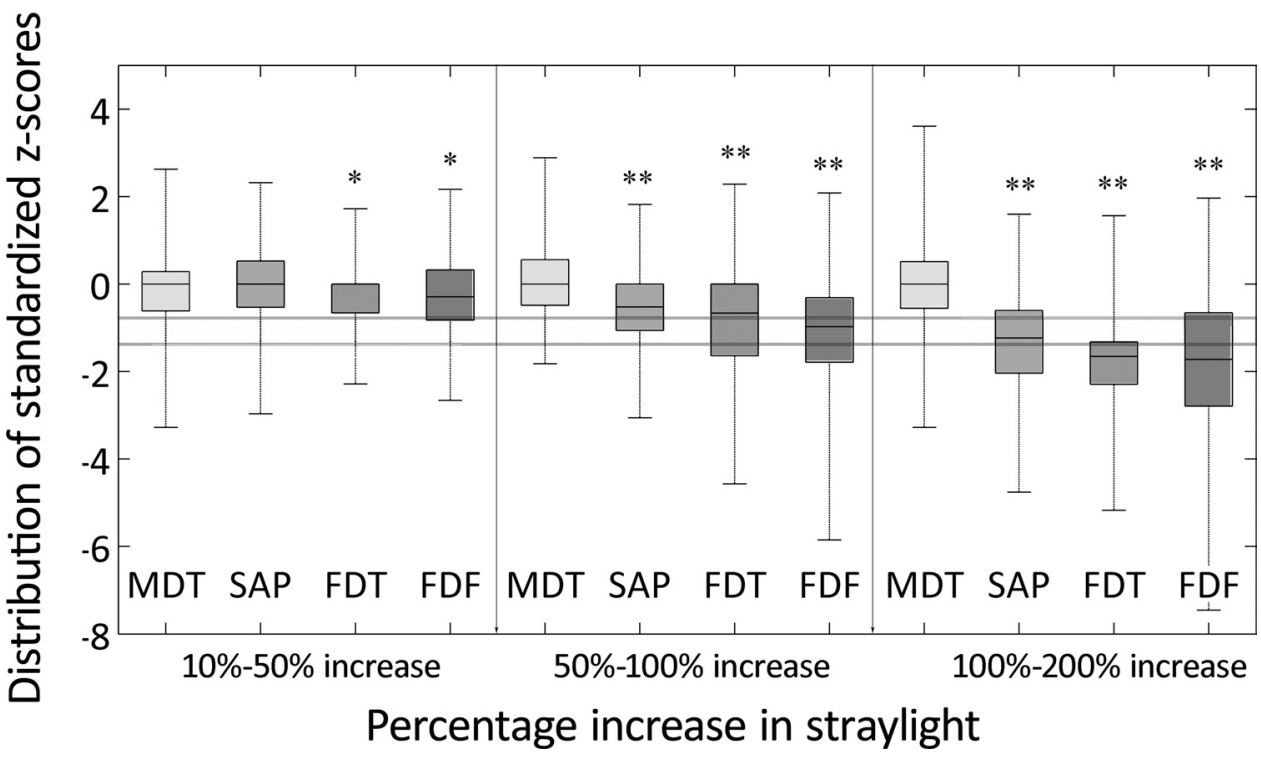

Figure 3. The effect of additional IOS on threshold measures. (A) Boxand-whisker plot of the TMS $z$-scores against the respective increase in intraocular straylight for the three subgroups 0 to $1.2,1.2$ to 1.6 , and 1.6 to $2.0 \mathrm{~dB}$. (B) Box-and-whisker plot of the distribution of TMS standardized $z$-scores for each instrument for three levels of IOS increase: $10 \%$ to $50 \%, 50 \%$ to $100 \%$, and $100 \%$ to $200 \%$. The line bisecting each box represents the median $z$-score. The upper and lower borizontal lines represent the $75 \%$ and $95 \%$ normative limits, respectively. ${ }^{*} P<0.01$ and ${ }^{* *} P<0.001$, those instruments affected by straylight.
In this study, we created IOS environments ranging from those associated with normal ageing to those encountered with significant cataract. This model allowed us to demonstrate the effect of various levels of IOS on perimetric thresholds and give a measure of the robustness of each instrument to such ocular imperfections. Throughout the range of IOS levels, the MDT thresholds remained relatively unchanged, whereas each of the other perimeters suffered greater loss in sensitivity with increasing IOS.

The use of these WOFs is an attempt to demonstrate not only the effects on modern perimetry of the additional IOS equivalent to those due to ageing, but also those due to significant cataract. Real cataracts have typically been graded according to four different parameters, depending on the location, color, density, and shape of the opacity within the lens. ${ }^{33}$ The WOFs used in this study are uniform-density filters and, as such, do not simulate the variety of forms of cataract. However, whatever the cataract type, it is the resulting IOS value that is most clinically meaningful and that best determines functional cataract severity. Thus, contrary to most previous studies, we have determined the individual IOS value for each simulation as the clinical grading measure.
The spatial characteristics of the test stimuli may account for the notable difference in robustness to the effects of IOS between the tests. Stimuli used in SAP, FDT, and FDF are contrast modulated, whereas the MDT stimulus remains at constant contrast, and the magnitude of displacement is modulated. So, whereas the MDT suffers an approximate $10 \%$ to $20 \%$ decrease in Michelson contrast with the most dense filter (fog 5), alternative contrast-modulated methods may have a $70 \%$ to $80 \%$ decrease in contrast at respective reference thresholds. The study result is therefore not surprising, given that contrast diminishes at the hands of optical imperfections arising from conditions such as cataract. ${ }^{24}$ To validate the findings, luminance was measured for the MDT stimuli with the densest of the WOFs (fog 5). The resultant stimulus energy was calculated (where energy is a composite of area and difference in luminance between the stimulus and the background, as described by Verdon-Roe et al.). ${ }^{30}$ Using the relationship between energy and MDT threshold, it is possible to calculate the expected threshold with and without the fog 5 WOF. This calculation estimated a decline in threshold to be less than 2 SD of the normal average. Notably, the effect reported was less. Anderson et al. ${ }^{33-35}$ found a similar result for robustness with 


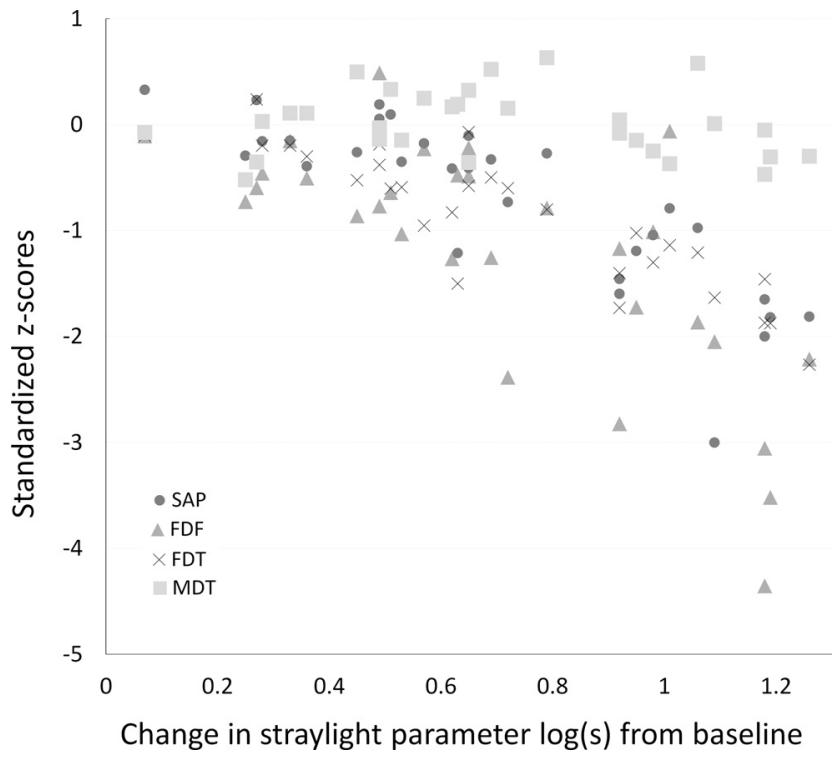

Figure 4. The change in TMS, expressed as standardized $z$-scores, with respect to the change in IOS. Each symbol represents a different instrument. Each marker shows a mean standardized $z$-score for one subject with one filter.

their peripheral grating-resolution test; notably it is a less contrast-dependent test.

In this study, the effect of IOS on SAP thresholds is similar to, but less marked than, that seen by Anderson et al.: 3.5 $\mathrm{dB} / \log (\mathrm{s})$ versus $4.9 \mathrm{~dB} / \log (\mathrm{s})$, respectively. ${ }^{25}$ This difference may be accounted for by the greater number of test locations in the present study $(n=52)$, as opposed to only four central test locations used in the analysis of Anderson et al. On examining the differences between central and peripheral locations with SAP and FDT from this study, it was found that peripheral locations were more affected by IOS $(P<0.05)$. A comparison of the FDT1 results reported by Anderson et al. can be made with the FDT matrix results from this study (while conscious that the stimulus size, testing procedures, and screen resolution of these instruments differ somewhat). The size of the effect reported here in terms of $z$-scores is slightly greater that those reported by Anderson et al. $(1.6 / \log (\mathrm{s})$ with FDT Matrix versus $1.3 / \log (\mathrm{s})$ with FDT1), and this may be due the additional peripheral locations and/or the smaller stimulus size of the FDT Matrix $\left(5^{\circ}\right.$ vs. $10^{\circ}$ patch of FDT1).

Clearly, the role of IOS is important when determining sensitivity in clinical perimetry. The implication is that either IOS should be measured in individuals at baseline and over time, or a form of perimetry little affected by IOS should be chosen. In this study it has been shown that increases in IOS, equivalent to those related to age, have measurable effects on SAP, FDT, and FDF thresholds (Figs. 3, 4). Knowing the effect of IOS on threshold values may enable tightening of normative ranges, as suggested by Anderson et al. ${ }^{25}$ This knowledge may also enable a methodology to correct for the diffuse loss of sensitivity resulting from cataract formation without also removing the diffuse loss component of glaucomatous damage. Tighter normal ranges would facilitate the identification of subtle sensitivity loss and provide greater confidence for diagnostic decisions. Therefore, further exploration of the influence of individual IOS measures on the normative databases of SAP, FDT, and FDF is needed.

\section{Conclusion}

There was little difference in the performance of FDT and SAP with respect to increases in IOS; both were significantly affected. FDF appears to be slightly more sensitive to increases in IOS than does SAP and FDT. There is little to no appreciable effect of IOS on MDT thresholds.

\section{Acknowledgments}

The authors thank John G. Flanagan (University of Waterloo, Waterloo and University of Toronto, Toronto, ON, Canada), Heidelberg Engineering, and Chris A. Johnson (University of Iowa, Iowa City, IA) for making the relevant limits of normality available; and Matilda Biba O'Neil, Angelos Sinapis, and Dimitrios Sinapis for their enthusiastic participation.

\section{References}

1. Quigley HA, Addicks EM, Green WR. Optic nerve damage in human glaucoma. III. Quantitative correlation of nerve fiber loss and visual field defect in glaucoma, ischemic neuropathy, papilledema, and toxic neuropathy. Arch Opbthalmol. 1982;100:135-146.

2. Harwerth RS, Carter-Dawson L, Shen F, Smith EL 3rd, Crawford ML. Ganglion cell losses underlying visual field defects from experimental glaucoma. Invest Ophthalmol Vis Sci. 1999;40:2242-2250.

3. Fraser S, Manvikar S. Glaucoma: the pathophysiology and diagnosis. Hosp Pharmacist (Lond). 2005;12:251-254.

4. Sample PA, Boynton RM, Weinreb RN. Isolating the color vision loss in primary open-angle glaucoma. Am J Opbthalmol. 1988;106: 686-691.

5. Artes PH, Iwase A, Ohno Y, Kitazawa Y, Chauhan BC. Properties of perimetric threshold estimates from Full Threshold, SITA Standard, and SITA Fast strategies. Invest Ophthalmol Vis Sci. 2002;43: 2654-2659.

6. Lam BL, Alward WL, Kolder HE. Effect of cataract on automated perimetry. Ophthalmology. 1991;98:1066-1070.

7. Hayashi K, Hayashi H, Nakao F, Hayashi F. Influence of cataract surgery on automated perimetry in patients with glaucoma. Am J Ophthalmol. 2001;132:41-46.

8. Membrey L, Fitzke FW. Effect of lens opacity on white-on-white perimetry, frequency doubling perimetry, and motion detection perimetry. In: Wall M, Wild JM, eds, Perimetry Update. The Hague, The Netherlands: Kugler Publications; 2000/2001:259266.

9. Tanna AP, Abraham C, Lai J, Shen J. Impact of cataract on the results of frequency-doubling technology perimetry. Opbthalmology. 2004;111:1504-1507.

10. Siddiqui M, Azuara-Blanco A, Neville S. Effect of cataract extraction on frequency doubling technology perimetry in patients with glaucoma. Br J Opbthalmol. 2005;89:1569-1571.

11. Werner JS. Development of scotopic sensitivity and the absorption spectrum of the human ocular media. J Opt Soc Am. 1982;72:247.

12. Pokorny J, Smith V, Lutze M. Aging of the human lens. Appl Opt. 1987;26:1437-1440.

13. Van Den Berg TJTP, Van Rijn LJ, Michael R, et al. Straylight effects with aging and lens extraction. Am J Opbthalmol. 2007; 144:358-363.

14. Said FS, Weale RA. The variation with age of the spectral transmissivity of the living human crystalline lens. Gerontologia. 1959;3:213.

15. Ruddock KH. The effect of age upon colour vision. II. Changes with age in light transmission of the ocular media. Vision Res. 1965;5:47.

16. Sample PA, Esterson FD, Weinreb RN, Boynton RM. The aging lens: in vivo assessment of light absorption in 84 human eyes. Invest Opbthalmol Vis Sci. 1988;29:1306-1311.

17. Van den Berg TJTP, Od F. Analysis of intraocular straylight, especially in relation to age. Optom Vision Sci. 1995;72:52.

18. de Waard $\mathrm{P}$, IJspeert J, van den Berg T, de Jong P. Intraocular light scattering in age-related cataracts. Invest Ophthalmol Vis Sci. 1992;33:618-625. 
19. Franssen L, Tabernero J, Coppens JE, van den Berg TJTP. Pupil size and retinal straylight in the normal eye. Invest Ophthalmol Vis Sci. 2007; 48:2375-2382.

20. de Wit GC, Franssen L, Coppens JE, van den Berg TJTP. Simulating the straylight effects of cataracts. J Cataract Refract Surg. 2006; 32:294-300.

21. Ijspreet JK, De Waard PWT, Van Den Berg TJ, De Jong P. The intraocular straylight function in 129 healthy volunteers: dependence on angle, age and pigmentation. Vision Res (Oxford). 1990 30:699-707.

22. Membrey L, Kogure S, Fitzke FW. A comparison of the effects of neutral density filters and diffusing filters on motion perimetry, white on white perimetry and frequency doubling perimetry. In Wall M, Wild JM, eds. Perimetry Update. The Hague, The Netherlands: Kugler Publications; 1998/1999:75-83.

23. Wood JM, Wild JM, Crews SJ. Induced intraocular light scatter and the sensitivity gradient of the normal visual field. Graefes Arch Clin Exp Ophthalmol. 1987;225:369-373.

24. Budenz DL, Feuer WJ, Anderson DR. The effect of simulated cataract on the glaucomatous visual field. Opbthalmology. 1993; 100:511-517.

25. Anderson RS, Redmond T, McDowell DR, Breslin KMM, Zlatkova MB. The robustness of various forms of perimetry to different levels of induced intraocular stray light. Invest Ophthalmol Vis Sci. 2009;50:4022-4028.

26. Franssen L, Coppens JE, van den Berg TJTP. Compensation com parison method for assessment of retinal straylight. Invest $O p b$ thalmol Vis Sci. 2006;47:768-776.
27. Anderson RS. The psychophysics of glaucoma: improving the structure/function relationship. Prog Retin Eye Res. 2006;25:79-97.

28. Anderson AJ, Johnson CA, Fingeret M, et al. Characteristics of the normative database for the Humphrey Matrix Perimeter. Invest Ophthalmol Vis Sci. 2005;46:1540-1548.

29. Quaid PT, Flanagan JG. Defining the limits of flicker defined form: effect of stimulus size, eccentricity and number of random dots. Vision Res. 2005;45:1075-1084.

30. Verdon-Roe GM, Westcott MC, Viswanathan AC, Fitzke FW, Garway-Heath DF. Exploration of the psychophysics of a motion displacement hyperacuity stimulus. Invest Opbthalmol Vis Sci. 2006; $47: 4847-4855$

31. Hill AB. Principles of Medical Statistics. 8th ed. London: The Lancet Limited; 1966.

32. Chylack L, Wolfe J, Singer D, et al. The Lens Opacities Classification System III. The Longitudinal Study of Cataract Study Group. Arch Opbthalmol. 1993;111:831-836

33. Anderson RS, Detkova P, O'Brien C. Effect of temporal frequency and contrast on peripheral grating resolution. Curr Eye Res. 1995; 14:1031-1033.

34. Anderson RS, Zlatkova MB, Beirne RO. The contrast sensitivity function for detection and resolution of blue-on-yellow gratings in foveal and peripheral vision. Opbthalmic Pbysiol Opt. 2002; 22:420.

35. Anderson RS. The selective effect of optical defocus on detection and resolution acuity in peripheral vision. Curr Eye Res. $1996 ; 15: 351$ 